\title{
Surface plasmon polariton modification in top-emitting organic light-emitting diodes for enhanced light outcoupling
}

\author{
Cornelius Fuchs, ${ }^{a}$ Tobias Schwab, ${ }^{a}$ Martin Wieczorek, ${ }^{a}$ Malte C. Gather,${ }^{a, b}$ Simone Hofmann, ${ }^{a}$ \\ Karl Leo ${ }^{a}$ and Reinhard Scholz ${ }^{a}$ \\ ${ }^{a}$ Institut für Angewandte Photophysik, Technische Universität Dresden, Georg-Bähr-Straße 1, \\ 01069 Dresden, Germany; \\ ${ }^{b}$ SUPA, School of Physics and Astronomy, University of St Andrews, St Andrews, KY16 9SS, \\ UK
}

\begin{abstract}
We report on the enhanced light outcoupling efficiency of monochrome top-emitting organic light-emitting diodes (OLEDs). These OLEDs incorporate a hole transport layer (HTL) material with a substantially lower refractive index $(\sim 1.5)$ than the emitter material or the standard HTL material $(\sim 1.8)$ of a reference device. This low-index HTL is situated between the opaque bottom metal contact (anode) and the active emission layer. Compared to an HTL with common refractive index, the dispersion relation of the surface plasmon polariton (SPP) mode from the opaque metal contact is shifted to smaller in-plane wavenumbers. This shift enhances the outcoupling efficiency as it reduces the total dissipated power of the emitter. Furthermore, the excitation of the coupled SPPs at the thin transparent metal top contact (cathode) is avoided by using an ultrathin top electrode. Hence, the coupling of the electroluminescence from the emitter molecules to all non-radiative evanescent modes, with respect to the emitter material, is reduced by at least a factor of two, additionally increasing the outcoupling efficiency.

Furthermore, for sufficiently high refractive index contrast the shift of the SPP at the anode/organic interface can lead to in-plane wavenumbers smaller than the wavenumber within the organic emitter layer and outcoupling of all excited modes by high index light extraction structures, e.g. microlens, seems feasible. In accordance to optical simulations, the external quantum efficiency is enhanced by about $20 \%$ for monochrome green emitting OLEDs with low refractive index HTL compared to a reference sample.
\end{abstract}

\section{INTRODUCTION}

Research towards improved organic light-emitting diode (OLED) efficiency is indispensable for widespread application in lighting and display technology. ${ }^{1,2}$ One measure for efficiency is the external quantum efficiency (EQE) $\eta_{\mathrm{EQE}}$, which is the ratio of emitted photons to injected charges during OLED operation. ${ }^{3}$ To quantify the EQE, it can be decomposed into a product of three factors $\eta_{\mathrm{EQE}}=\gamma \eta_{\text {rad }} \eta_{\text {out }},{ }^{4,5}$ where the first factor $\gamma$ denotes the electrical efficiency and charge carrier balance of the OLED. For state-of-the-art OLEDs $\gamma$ approaches unity, relying on the pin-concept with doped charge carrier transport layers embedding intrinsic blocking and emission layers. ${ }^{6}$ The second factor $\eta_{\text {rad }}$ describes the amount of electron-hole pairs converted into excitons and the probability of radiative recombination for this exciton. ${ }^{5}$ Due to exciton harvesting techniques, ${ }^{7}$ this factor exceeds 0.7 for many emission wavelengths. Thus, the outcoupling efficiency $\eta_{\text {out }}$ defined by the outcoupled electromagnetic power divided by the total power provided by the source often becomes the limiting factor for high efficiency. For common top-emitting OLEDs radiating the light directly from the organic layers through a thin transparent electrode layer, this factor rarely surpasses $0.3 .^{2,8,9}$ In top-emitting OLEDs, light-emission through a substrate which leads to further radiation loss is avoided, and thus the above-mentioned limit is a typical upper boundary for OLEDs in general. The limitation is caused by two interrelated effects. Firstly, the refractive index of the organic material $n_{\text {org }} \simeq 1.8$ prevents large amounts of radiation to be outcoupled to air due to total internal reflection (TIR). ${ }^{10}$ Furthermore, due to the thin organic layers, the reflections at the materials

Further author information: (Send correspondence to C.F.)

C.F.: E-mail: cornelius.fuchs@iapp.de, Telephone: +49 35146336446

Organic Light Emitting Materials and Devices XVIII, edited by Franky So, Proc. of SPIE Vol. 9183, 91831Z

(C) 2014 SPIE · CCC code: 0277-786X/14/\$18 · doi: 10.1117/12.2061585 
interfaces, especially from TIR, are superposed coherently, leading to resonance effects of an optical microcavity. Thus, the excitation of radiation is pronounced for discrete modes of the optical microcavity which are mostly non-radiative for far field emission. ${ }^{5}$ As the refractive index of the organic materials, in particular the one of the emitter material, cannot be changed (lowered) arbitrarily to decrease the limitation from TIR, the optical microcavity is optimized to reduce losses to the discrete modes of the optical microcavity. For common OLEDs these divide into wave guide modes (WGs) and excitations of surface plasmon polaritons (SPPs). Waveguides cannot be avoided by OLED optimization because an efficient OLED cavity needs to form an optical microcavity with at least one WG. ${ }^{5,11,12}$ Although for top-emitting OLEDs this WG mode is partially radiating to the far field (leaky WG), ${ }^{13}$ further optimizations for such cavities include capping layers ${ }^{14}$ introducing higher order bound WG modes. Using metallic electrodes in the OLEDs leads to the excitation of SPPs. While the SPP forms at the metallic/dielectric interface, it can be suppressed by enlarging the distance of the emitter material to the metal, which in turn increases the number of WG modes within the cavity. Thus, such high order top-emitting devices remain less efficient than the corresponding first order device. ${ }^{1,15}$

In this paper, we explain an approach to modify the SPPs dispersion relation by incorporating a low refractive index material next to the opaque bottom contact. Thus the power dissipation characteristics of emitters embedded in this optical microcavity is changed. We present a detailed analysis of the physical model and demonstrate experimental data showing an enhancement of about $20 \%$ EQE for green top-emitting OLEDs, in correspondence to the results of optical simulations.

\section{THEORETICAL CONCEPT}

Throughout this paper, we will denote the growth direction perpendicular to the plane of the substrate as the $z$-direction. Surface plasmon polaritons correspond to quanta of electromagnetic field propagating along a metal/dielectric interface caused by the coupling of charge density oscillations at the metal surface (surface plasmon) to the electromagnetic field. ${ }^{10,16}$ Within stratified media with dielectric function $\epsilon$, the electromagnetic field of optical modes, and in particular the SPP, can be modeled as an electromagnetic wave with wave vector components $k_{x}$ and $k_{z}$ following $\epsilon k_{0}^{2}=k_{x}^{2}+k_{z}^{2}$. As the SPP excitation is bound to the interface $\left|k_{x}\right|>\left|\epsilon^{1 / 2} k_{0}\right|$, $k_{z}$ becomes imaginary for the SPP even though the dielectric function of the dielectric medium remains real. We assume a metal with dielectric function $\varepsilon_{\text {metal }}$ and a dielectric medium with dielectric function $\varepsilon_{\text {dielectric }}$ to extend over the lower and upper half space with the interface being at $z=0$. By using the boundary conditions at the metal/dielectric interface, derived from Maxwell's equations, the dispersion relation for such opaque metal SPPs (oSPPs) within the aforementioned geometry reads ${ }^{16}$

$$
E=k_{x} c \hbar \sqrt{\frac{\varepsilon_{\text {metal }}+\varepsilon_{\text {dielectric }}}{\varepsilon_{\text {metal }} \varepsilon_{\text {dielectric }}}},
$$

where $E$ denotes the energy of the oSPP, $c$ the speed of light in vacuum, and $\hbar$ the reduced Planck constant. Figure 1 visualizes the dispersion relation for two different dielectric materials. For a material with common

dielectric constant $\varepsilon_{\text {high }}$ the real part $n_{\text {high }}=\Re\left[\epsilon_{\text {high }}^{1 / 2}\right]$ of the refractive index is assumed to be 1.75 . The second medium is assumed to have a lower real part of the refractive index, $n_{\text {low }}=1.525$. For all dielectric materials the imaginary part of the refractive index, known as the extinction coefficient, is assumed to be negligible. The dielectric function of the metal is modeled to be the one of aluminum ${ }^{5}$ within the visible frequency range. Thus a Drude model fit is used with an energy equivalent of the plasma frequency $E_{\mathrm{p}}=12.23 \mathrm{eV}$ and a background dielectric response of $\epsilon_{\mathrm{b}}=-0.943$. Charge carrier damping effects within the metal, corresponding to imaginary part of the dielectric function $\Im[\epsilon]$, were neglected within the range of interest. In Fig. 1, we observe a shift of the dispersion relations for the high and low refractive index dielectric material oSPPs, which are depicted as dashed lines. The magnitude of this shift $\Delta k_{x}=k_{x}$, low $-k_{x}$, high is $\Delta k_{x, 1.8 \mathrm{eV}}=-2.32 \mu \mathrm{m}^{-1}$ for the lowest energy of interest, to $\Delta k_{x, 2.7 \mathrm{eV}}=-3.64 \mu \mathrm{m}^{-1}$ for the highest energy of interest.

At the emission maximum of our green phosphorescent iridium complex (cf. Sec. 3), the decay lengths ${ }^{17}$ $l_{z}=1 /\left|k_{z}\right|$ of the oSPPs electromagnetic field into the dielectric medium can be obtained as $l_{z, \text { low }, 2.43 \mathrm{eV}} \simeq 170 \mathrm{~nm}$ and $l_{z}$, high, $2.43 \mathrm{eV} \simeq 130 \mathrm{~nm}$ respectively. As we neglected the absorption of the oSPP in $x$-direction (no charge carrier damping), these values give a lower boundary and thus for real devices the decay lengths normal to the interface are expected to be larger. However, even for this lower limit, these decay lengths are within the same 


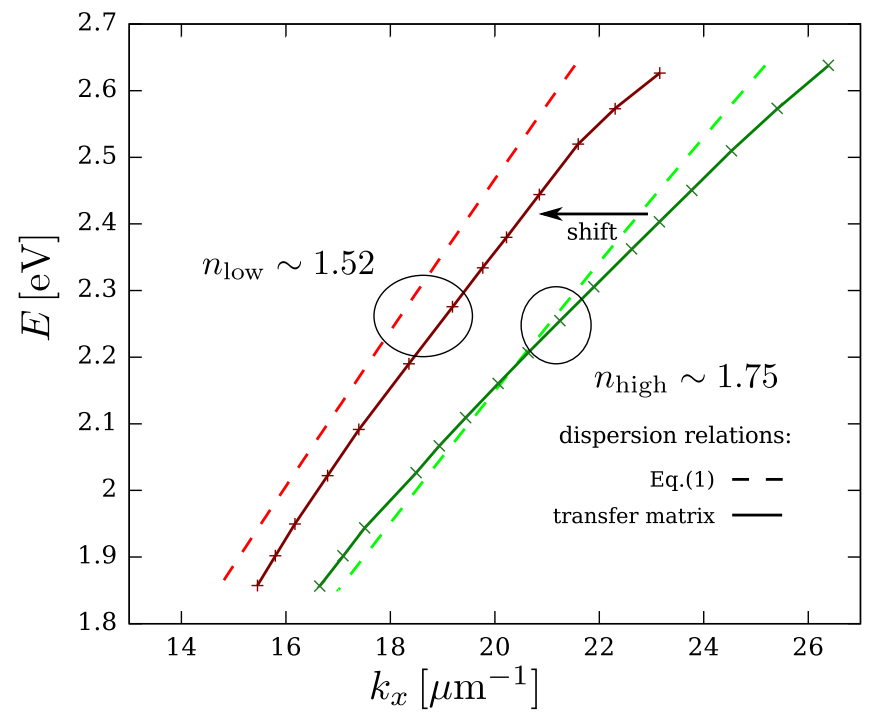

Figure 1. Calculated dispersion relations for oSPPs from Eq. (1) (dashed lines) and from resonances in the transfer-matrix (solid lines). For a simple model, two dielectric media with $n_{\text {low }}=1.525$ and $n_{\text {high }}=1.75$ are taken into account attached to a metal with a Drude dielectric function with $E_{\mathrm{p}}=12.23 \mathrm{eV}$ and $\epsilon_{\mathrm{b}}=-0.943$. For the transfer-matrix method the refractive indices according to Fig. 5 were used.

order of magnitude as the distance between emitter and the opaque metal contact as well as the total optical thickness of the OLED microcavity. Hence, this simple analysis based on Eq. (1) may not be accurate enough to describe the shift of the oSPPs dispersion relation within the OLED microcavity. Thus we will need to calculate the oSPPs dispersion relation by taking into account the multiple field reflections within the complete OLED microcavity stack. Therefore we calculate the transfer-matrix for the TM-polarized magnetic field amplitudes for the complete optical microcavity of the OLED. ${ }^{13,18}$ The dispersion relations of optical modes supported by this microcavity are obtained from the roots of the transfer-matrix elements due to the resonant transmission enhancement ${ }^{13,16-19}$ for optical modes within the cavity. The device with the common high refractive index organic HTL next to the opaque metal layer will be referred to as Device A whereas the geometry using the low refractive index HTL is called Device B. For details on the layer sequence, the reader is referred to the experimental section. These results for the dispersion relation for the low refractive index adjacent layer and the high refractive index layer are also depicted in Fig. 1 as solid lines. The observed shift is now corresponding to $\Delta k_{x, 1.8 \mathrm{eV}}=-1.21 \mu \mathrm{m}^{-1}$ for low photon energies and to $\Delta k_{x, 2.7 \mathrm{eV}}=-2.98 \mu \mathrm{m}^{-1}$ for high energies. We note that also within a multilayer geometry we observe a shift for the oSPP dispersion relation similar to the simple model. The deviations for the absolute values of the shifts and for the dispersion relation positions are caused by taking into account measured refractive indices for all materials, included non-vanishing $\Im[\epsilon]$ for the metal layer and the finite geometry. Even though the shift decreases, the physical nature of the effect remains the same and can be used to modify the oSPPs dispersion relation in OLEDs.

By using established numerical methods, ${ }^{5,20}$ we are able to simulate the expected power dissipation from emitting dipole sources into stratified microcavities. In these models the light-emitting sources are represented by an arbitrary thin layer of polarization within the OLED microcavities which is driven coherently by the electromagnetic field. ${ }^{21}$ The power dissipation $F$ represents a measure of how likely it is that the emitter will emit radiation with a particular in-plane wavenumber (emission angle), normalized to the power dissipation in an infinite medium of same dielectric function, and thus represents the coupling between emitter and geometry. The outcoupling efficiency $\eta_{\text {out }}$ is calculated for each emitted wavenumber $k_{0}$ (wavelength $\lambda=2 \pi k_{0}^{-1}$ ) as the outcoupled power $K_{\text {out }}=2 \int_{0}^{k_{x} \text {, crit }} \mathrm{d} k_{x} k_{x} F_{\text {out }}\left(k_{x}\right)$ divided by $K$, which describes the total source provided power for a given wavenumber normalized to the power provided by a source within an infinite dielectric medium $K=2 \int_{0}^{\infty} \mathrm{d} k_{x} k_{x} F\left(k_{x}\right) .{ }^{5}$ Hereby the in-plane wavenumber limit for light extraction is given by TIR and reads 
as $k_{x, \text { crit }}=\frac{n_{\text {air }}}{n_{\text {active }}} k_{0}$. Evanescent modes along the growth direction $z$ can be represented by electromagnetic plane waves with in-plane wavenumbers $k_{x}>k_{0} n_{\text {active. }}$. Thus the real space electromagnetic field amplitude decreases exponentially within media with real parts of the refractive index larger or equal to the refractive index of the emitter material $n_{\text {active }}$ or metals. Because of the decreasing field the mode is called non-radiative or evanescent. Within this model, it is clear that the excitation of the polarization for evanescent modes must vanish for increasing in-plane wavenumbers $k_{x}$. If we use a low refractive index material to shift the oSPP to smaller in-plane wavenumbers, the power dissipation can drop faster to zero. The simulated power dissipation for Device A and Device B is shown in Fig. 2 (a). For these simulations, both devices exhibit a transparent $20 \mathrm{~nm}$ silver top contact and are optimized regarding the HTL, ETL and capping layer thickness for maximized light outcoupling. The photon energy was set to $2.43 \mathrm{eV}$ corresponding to the emission maximum of the used emitter material (cf. Fig. 5(a)). Furthermore, in Fig. 2 (a) the limits of outcoupled radiation and WG modes are highlighted by solid vertical lines. The corresponding outcoupling efficiencies for Device A and B exhibiting
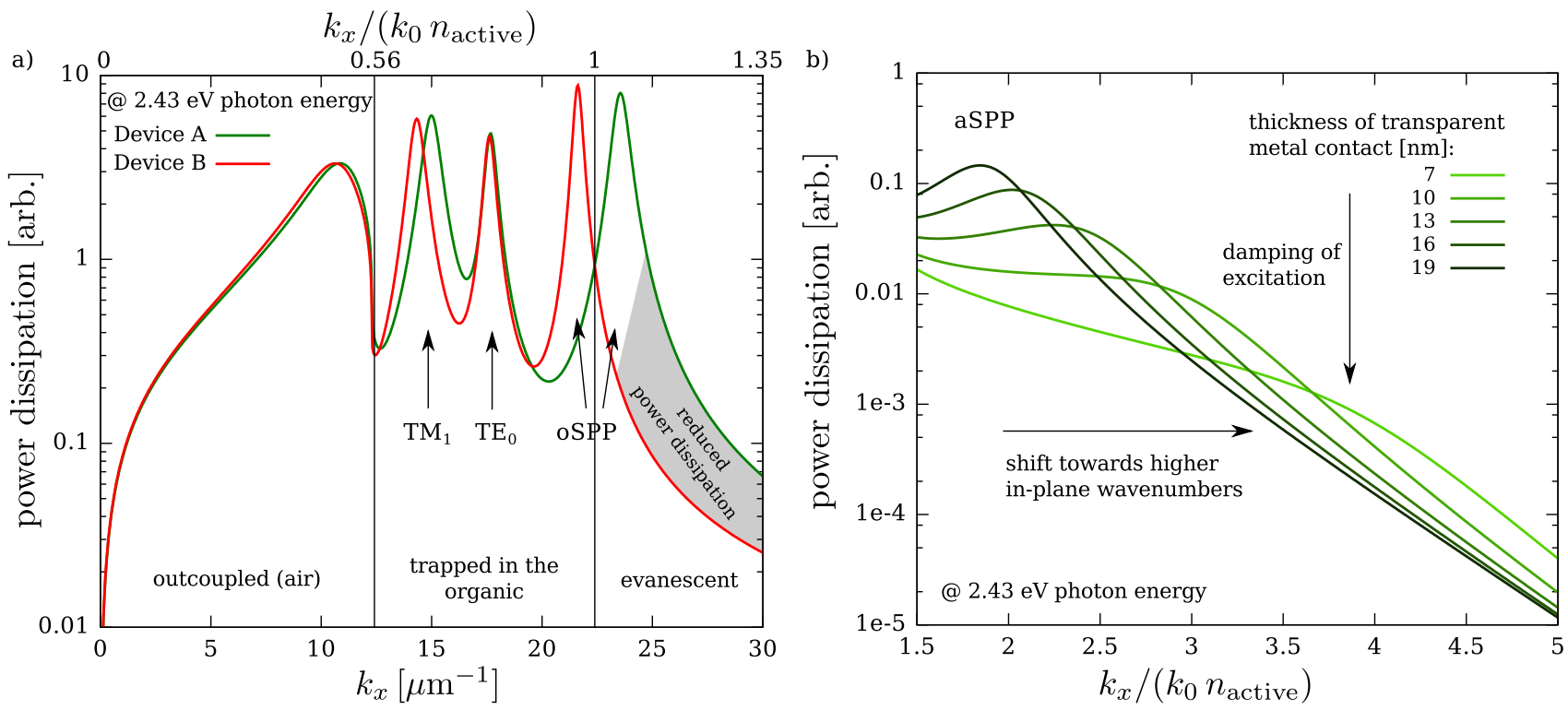

Figure 2. a) Power dissipation for Device A and Device B, at $2.43 \mathrm{eV}$ photon energy, corresponding to the emission maximum of the green phosphorescent iridium complex $\operatorname{Ir}(\mathrm{ppy})_{3}$. Compared to Device A, the power dissipation is lowered for the evanescent modes of Device B due to the low refractive index of the HTL of Device B. Thus the total dissipated power is decreased, enhancing the outcoupling efficiency. b) Power dissipation for different thicknesses of the transparent metal top contact. The peaks represent the aSPP position. By decreasing the metal thickness the aSPP mode is shifted to larger in-plane wavenumbers. Thus, its excitation is exponentially decreased, leading to an enhanced outcoupling efficiency.

a $20 \mathrm{~nm}$ transparent silver contact are calculated to $\eta_{\text {out, }}, 20=27.1 \%$ for the high refractive index HTL and $\eta_{\text {out, B } 20}=33.1 \%$ for the low refractive index HTL. This corresponds to an enhancement of $22 \%$. From Fig. 2 (a) we see that the outcoupling efficiency is enhanced from as the power dissipated in the evanescent regime is reduced due to the shift of the oSPP to smaller wavenumbers $k_{x}$. We like to emphasize that the WG modes are only slightly modified in position and unchanged in intensity along with the power dissipation to the outcoupled radiation. This was expected as the total optical thickness of Device A and B is the same for both devices. Thus the enhancement of the outcoupling efficiency is due to the change of total emitted power and not attributed to a change of outcoupled power.

To enhance this effect even more we will use a recently developed wetting layer electrode ${ }^{22,23}$ as transparent top contact. These electrodes consist of a very thin layer of metal deposited onto a seed metal thus forming a closed planar film. This enables high transparencies combined with very low sheet resistances by avoiding the complicated deposition process for competing electrode materials like transparent conductive oxides. Moreover, the combined thickness of metal layer and seed material does not exceed $10 \mathrm{~nm}$, proving beneficial for the properties of the SPPs excited at these metal interfaces. For such a thin metal film the SPPs excited at either 
surface of the thin film will couple to a symmetric (sSPP) and an antisymmetric state (aSPP), regarding the $z$-component of the electrical field. ${ }^{16}$ Within the visible frequency range the SSPP is located at smaller in-plane wavenumbers than the aSPP. However, it does not couple to emissive dipoles within the cavity ${ }^{18,24}$ due to the strong localization in $x$ - and $z$-direction, and is thus omitted in this discussion. The aSPP is excited by emissive dipoles within the cavity for TM-polarized radiation due to the finite electromagnetic field amplitude at the emitter location. However, this interaction is quickly reduced with decreasing metal layer thickness as the dispersion relation of the aSPP shifts to higher in-plane wavenumbers, thus improving the localization around the interface. The reduced influence of the aSPP to the emitting dipoles can be seen in Fig. 2 (b), where the power dissipation for aSPPs of different metal thicknesses (rest of the device is same as Device A) is shown in detail. The outcoupling efficiencies for both types of HTL are enhanced by about $12 \%$ by reducing the transparent silver layer thickness from $20 \mathrm{~nm}$ to $7 \mathrm{~nm}$, whereas $\eta_{\text {out }}$ for the high index HTL devices increases from $\eta_{\text {out }, \mathrm{A}, 20}=27.1 \%$ to $\eta_{\text {out }, \mathrm{A}, 7}=30.2 \%$ and respectively for the low refractive index HTL from $\eta_{\text {out }, \mathrm{B}, 20}=33.1 \%$ to $\eta_{\text {out }, \mathrm{B}, 7}=37.1 \%$. Additionally, these ultrathin metal layers formed by the wetting layer approach grow as a very smooth film. ${ }^{22}$ Thus, within the real device, the coupling of electromagnetic fields to the SPP caused by surface roughness ${ }^{17}$ decreases. By combining the oSPP shift and the thin metal layer, the outcoupling efficiency increases significantly by $37 \%$ from the outcoupling efficiency for the non-shifted, thick transparent metal device $\eta_{\text {out, }, 20}$ to $\eta_{\text {out, B, } 7}$.

However, we focus on the oSPP modification. To fully show effect of the oSPP shift, the simulated power dissipation spectra for all relevant photon energies (cf. the photoluminescence spectrum of the green emitter in the appendix Fig. 5 (a) ) are shown in Fig. 3. Figure 3 (a) gives a detailed view on the power dissipation of Device A and for Device B the simulation results are shown in Fig. $3(\mathrm{~b})$. The refractive indices and extinction coefficients

a)

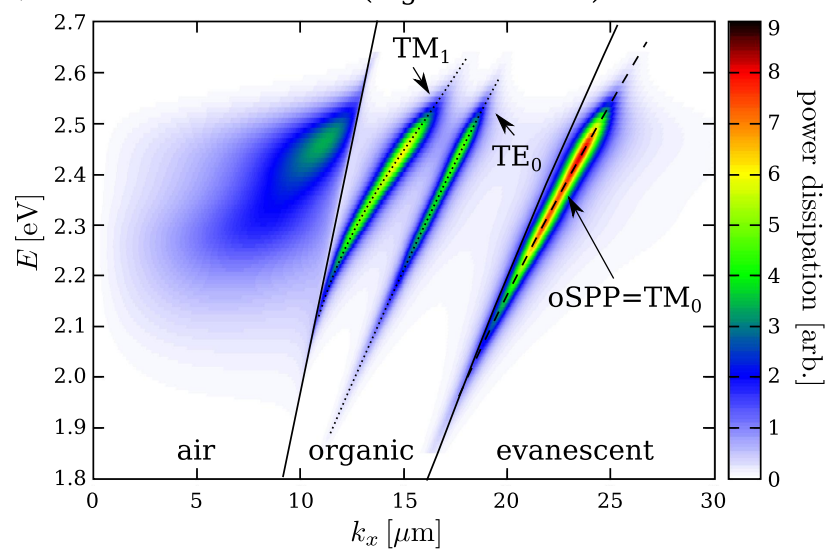

b)

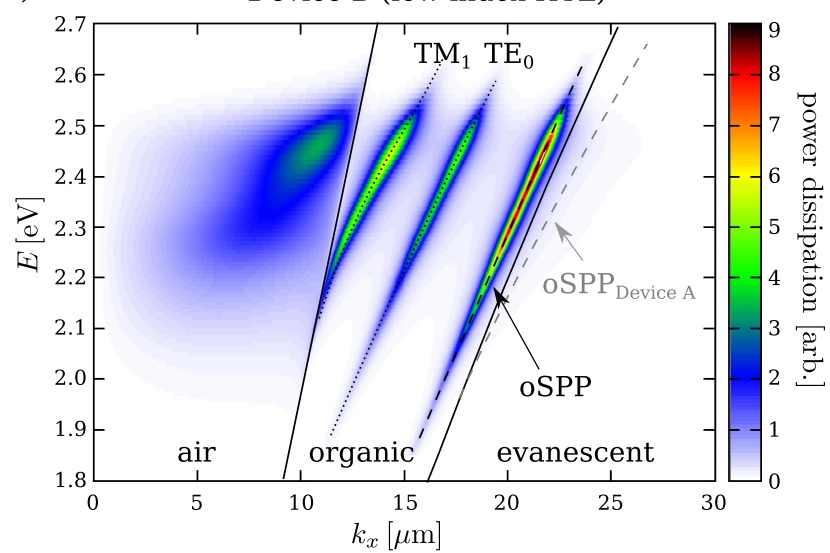

Figure 3. Simulated power dissipation spectra for a) Device A and b) Device B. The solid lines highlight the radiative boundaries for air and the organic media. The dispersion relations for the WG (dotted) and oSPP (dashed) modes are also highlighted. Due to the low refractive index of the HTL for Device B, a shift for the oSPPs dispersion relation is observed, corresponding to the previous results. The oSPPs dispersion of Device A is shown in (b) as gray dashed line for reference.

underlying the simulation are given in the appendix in Fig. 5. The oSPP/WG modes occurring in these devices are highlighted by dashed/dotted lines. Additionally, the in-plane wavenumbers corresponding to the air light line $k_{0}$ and the organic light line $k_{0} n_{\text {active }}$ are represented by solid lines which limit the radiative modes in air and organic. We observe a shift for the oSPP mode of $\Delta k_{x, 1.8 \mathrm{eV}}=-1.12 \mu \mathrm{m}^{-1}$ to $\Delta k_{x, 2.7 \mathrm{eV}}=-2.79 \mu \mathrm{m}^{-1}$ which is in good agreement with the results previously obtained (cf. Fig 1). To guide the eye the oSPPs dispersion for Device A, (oSPP Device A) is shown as gray dashed line in Fig. 3 (b). The simulation is shown up to in-plane wavenumbers $k_{x}$ equivalent to $1.35 k_{0} n_{\text {active }}$ at $510 \mathrm{~nm}$ emission wavelength. Thus the dispersion relation of the aSPPs is not present in Fig. 3 for high energies due to the limit in $k_{x}$ (compare their positions in Fig. 2 (b)), but for low energies due to the vanishing contribution to the power dissipation (compare the aSPP intensity in Fig. 2 (b) ). However, for the calculation of the outcoupling efficiency the power dissipation is taken into account up to $5.5 k_{0} n_{\text {active }}$, thus including these very weak contributions. Similar to the previous calculations for the thick 
transparent metal layer both devices were optimized for maximized outcoupling efficiency. As previously this optimization leads to devices with same optical thickness, which means both devices support the same amount of WG modes. The enhancement of the outcoupling efficiency attributed to the oSPP shift can be quantified to a factor of 1.23 from $\eta_{\text {out }, \mathrm{A}, 7}=30.2 \%$ for Device A to $\eta_{\text {out }, \mathrm{B}, 7}=37.1 \%$ for Device B, corresponding to the enhancement for the thick transparent contact.

In conclusion, by using low refractive index materials as adjacent layers to the opaque metal the oSPP dispersion relations can be shifted to lower in-plane wavenumbers efficiently decreasing the power dissipation for evanescent excitations. As this decreases the total dissipated power the outcoupling efficiency is enhanced. The excitation at larger in-plane wavenumbers, e.g. the aSPP, are avoided by using an ultrathin metal top-contact. This compresses the power dissipation further in phase space thus, enhancing the outcoupling efficiency even more.

\section{EXPERIMENTAL}

To validate our theoretical concept concerning the shift of the oSPP, we fabricated two OLEDs, a reference Device A and an OLED with low refractive index HTL, Device B. Both devices consist of an opaque aluminum anode of $100 \mathrm{~nm}$ thickness followed by a hole transport material. For Device A this was $2,2^{\prime}, 7,7^{\prime}$ tetra(N,N-ditolyl)amino-9,9-spiro-bifluorene (Spiro-TTB) doped with 4 wt.\% of 2,2 -(perfluoro-naphthalene-2,6diylidene)dimalononitrile (F6-TCNNQ) with a thickness of $49 \mathrm{~nm}$. In Device B Poly(3,4-ethylenedioxythiophene)poly(styrenesulfonate) (PEDOT:PSS) (55 nm) was used as HTL exhibiting significantly lower refractive index than Spiro-TTB (cf. Fig. 5 in the appendix). Following onto these transport layers, the OLEDs exhibit an electron blocking layer (EBL) of 2,2',7,7'-Tetrakis-(N,N-diphenylamino)-9,9'-spirobifluorene (Spiro-TAD) (10 nm), a double emission layer (EML) of 4,4',4"-tris (carbazol-9-yl)-triphenylamine (TCTA) $(6 \mathrm{~nm})$ as one host material and 2,2',2"-(1,3,5-Phenylen)tris(1-phenyl-1H-benzimidazole) (TPBi) $(12 \mathrm{~nm})$ as a second both doped with $8 \mathrm{wt} \%$ of the phosphorescent green emitter Tris(2-phenylpyridine) iridium(III) ( $\left.\operatorname{Ir}(\mathrm{ppy})_{3}\right)$ were used. The OLED is completed by a hole blocking layer (HBL) consisting of 4,7-diphenyl-1,10-phenanthroline (BPhen) (10 nm), an electron transport layer (ETL) of BPhen doped with cesium $(43 \mathrm{~nm})$, the ultrathin top electrode comprising a $2 \mathrm{~nm}$ seed gold layer and $7 \mathrm{~nm}$ of silver, and an organic capping layer of N,N'-Di(naphthalen-1-yl)-N,N'-diphenylbenzidine $(\alpha$-NPD) $(89 \mathrm{~nm})$. In total two samples, each with four independent operating pixels, were fabricated for each device.

In this layer sequence the anode is deposited at first by physical vapor deposition in an UHV tool (Kurt J. Lesker Co.) at a base pressure of $10^{-7}-10^{-8}$ mbar. Afterwards the samples were treated by an argon plasma at $10^{-1} \mathrm{mbar}$ for $10 \mathrm{~min}$ to enhance the surface adhesion properties of the aluminum surface to the PEDOT:PSS in Device B. The PEDOT:PSS (Clevios P AI4083) is then spin-coated onto the substrate of Device B at $2400 \mathrm{rpm}$ to achieve a film thickness of $55 \mathrm{~nm}$, followed by a baking step of $15 \mathrm{~min}$ at $110^{\circ} \mathrm{C}$. Next the substrates were transfered to vacuum and again heated out for $1 \mathrm{~h}$ at $110^{\circ} \mathrm{C}$. Subsequently, the HTL of Device A and all other layers were deposited under UHV conditions similar to the deposition conditions for the anode. After the last deposition step, the devices were encapsulated with a glass lid under nitrogen atmosphere. The current-voltageluminance $(I-V-L)$ characteristics of the two devices were determined by a source measure unit (Keithley 2400) and a calibrated spectrometer (Instrument Systems CAS140) providing the spectral radiant intensity. Angledependent measurements of the spectral radiant intensity for emission angles $\theta\left(0^{\circ} \leq \theta \leq 90^{\circ}\right)$ were performed with a spectro-goniometer and a calibrated miniature spectrometer (USB 4000, Ocean Optics Co.). The EQE is calculated from the $I-V-L$ and the angle-dependent emission spectra of the OLEDs.

\section{RESULTS AND DISCUSSION}

The measured $I-V-L$ for Device A and B are shown in Fig. 4 (a). We note an increased leakage current for Device B of about one order of magnitude. This is caused by the deposition of PEDOT:PSS ex-situ, which is likely to introduce impurities. In forward direction, beyond the onset voltage of the OLEDs, the slope of the $j-V$ curve is slightly smaller for Device B. We attribute this effect to the fact that by changing the hole transporting material from Spiro-TTB:F6-TCNNQ to PEDOT:PSS the charge injection barriers from the aluminum to the $\mathrm{HTL}^{25}$ and from the HTL to the $\mathrm{EBL}^{26}$ will change. The PEDOT doped with the polystyrenesulphonic acid 

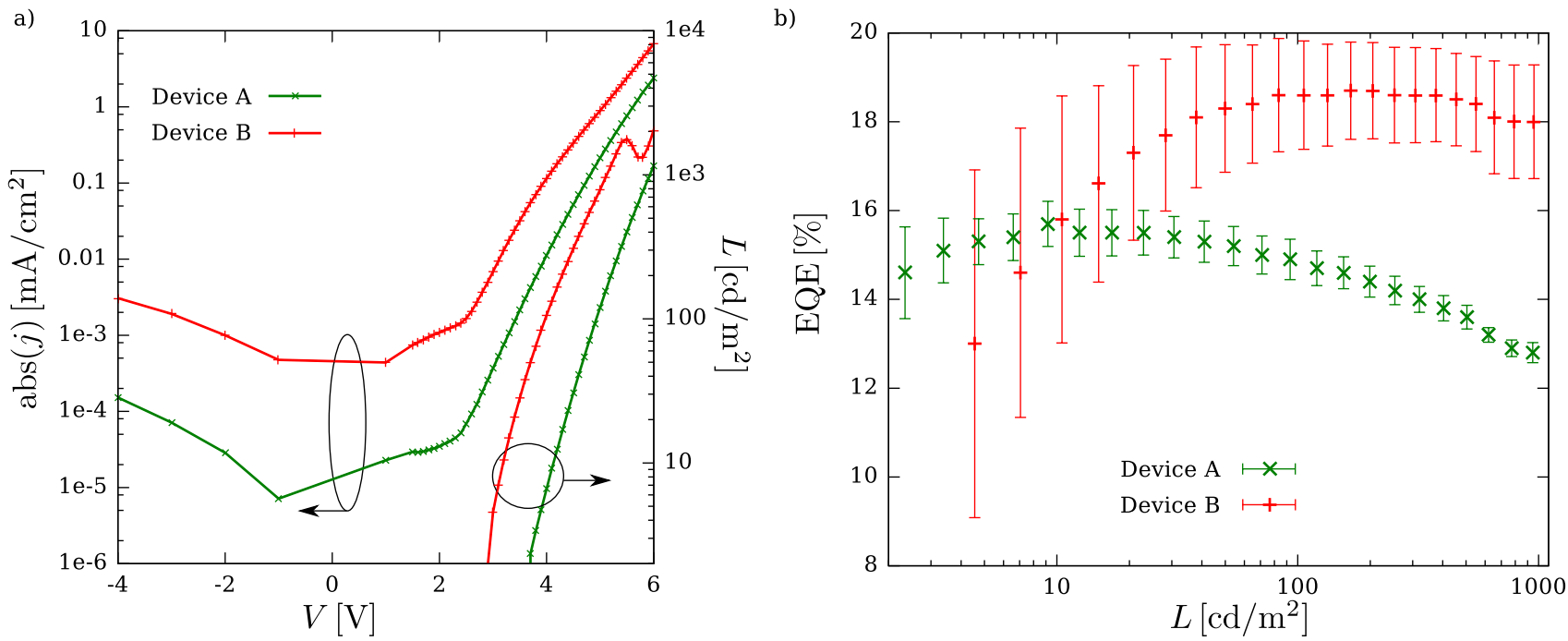

Figure 4. a) Absolute values of the current density $j$ and corresponding luminance $L$ as a function of voltage $V$, for Device A and B. b) External quantum efficiency (EQE) as a function of luminance. The EQE is calculated from the angle-dependet emission spectra. We notice a maximum EQE enhancement of about $20 \%$ from $15.7 \%$ for Device A to $18.7 \%$ for Device B. The errors are calculated from the standard error of the pixel deviations.

(PSS) forms a corrosive aqueous PEDOT:PSS dispersion, ${ }^{26}$ which enhances the substrate adhesion but leads to enhanced interlayer formation on the metal surface. ${ }^{25}$ Thus, the aluminum oxide injection barrier at the anode/HTL interface will be more pronounced compared to the one for UHV processed aluminum and SpiroTTB, leading to the changes of the $j-V$ characteristics measured on Device B with respect to Device A. The luminance in forward direction is depicted on the right scale of Fig. 4 (a) and shows the typical linear increase with current. For current densities exceeding $\sim 2.5 \mathrm{~mA} / \mathrm{cm}^{2}$, the luminance for Device B follows an s-shape. This behavior could be also observed for Device A, but as a similar current density is reached at higher voltage, this region is not included in Fig. 4 (a). We attribute this effect to the bottom electrode, as for both devices the substrates were taken out of the vacuum and plasma treated to achieve comparable oxidation of the aluminum. A detailed explanation however is beyond the scope of this paper. Moreover, the charge carrier balance is optimized for Device A and introducing a different HTL will yield a different EQE roll-off behavior for Device B, as can be seen in Fig. 4(b). Thus, we will not compare the EQE for Device A and B at the same current densities, but instead at their respective maxima. From Fig. 4 (b) we observe an increase for the maximum EQE of about $20 \%$ from $\eta_{\mathrm{EQE}, A}=15.7 \pm 0.6 \%$ for Device A to $\eta_{\mathrm{EQE}, B}=18.7 \pm 1.6 \%$ for Device B. The increase in measurement errors, calculated from the standard error, are due to the spin-coating process for the HTL of Device B, which leads to higher sample to sample/pixel to pixel variations. As the intrinsic layers (EBL, EML, HBL) for Device A and B are the same, we assume the radiative efficiency $\eta_{\text {rad }}$ to be equal for both devices. Thus, within the error of measurement the enhanced outcoupling efficiency, predicted by the simulations, can be found in the enhancement of the EQE combined with a minor decrease in electrical efficiency. To support this statement, we fabricated several OLEDs of the type of Device B with varying HTL thickness. The maximum EQEs obtained for these devices were observed for all HTL thicknesses at comparable brightness and are given in Table 1. Along with the EQEs, the calculated outcoupling efficiencies from the fitted emission spectra are shown. Determining the outcoupling efficiency and measuring the EQE allows to obtain the factor $\gamma \eta_{\text {rad }}$ of the OLED,${ }^{5}$ which for the same HTL material should remain constant. These quantities are also shown in Table 1 accompanied by the enhancement factors for the OLEDs incorporating PEDOT:PSS as HTL in comparison to Device A. We observe the factor $\gamma \eta_{\text {rad }}$ can be treated as a constant within the range of the measurement error. As the small variation of HTL thickness should not influence the electrical or internal radiative efficiencies, This is further evidence that the EQE enhancement is due to the improved outcoupling efficiency resulting from the compression of the phase space region into which power is dissipated. 
Table 1. Summarized calculated outcoupling efficiencies from fitted emission spectra, measured maximum EQE, error of maximum EQE, brightness $L_{\max \left(\eta_{\mathrm{EQE}}\right)}$ where the EQE was measured, calculated factors $\gamma \eta_{\mathrm{rad}}$, and enhancement factors compared to Device A for OLEDs of type Device B with varying HTL thicknesses.

\begin{tabular}{|c|c|c|c|c|c|c|}
\hline HTL thickness $[\mathrm{nm}]$ & $\eta_{\text {out }}[\%]$ & $\eta_{\mathrm{EQE}}[\%]$ & $\Delta \eta_{\mathrm{EQE}}[\%]$ & $L_{\max \left(\eta_{\mathrm{EQE}}\right)}\left[\mathrm{cd} / \mathrm{m}^{2}\right]$ & $\gamma \eta_{\mathrm{rad}}$ & enhancement factor \\
\hline 47 & 32.9 & 16.3 & 0.4 & 200 & 0.50 & 1.04 \\
51 & 34.7 & 17.7 & 1.5 & 160 & 0.51 & 1.13 \\
55 & 35.4 & 18.7 & 1.6 & 170 & 0.53 & 1.19 \\
63 & 34.5 & 17.6 & 1.7 & 230 & 0.51 & 1.12 \\
\hline
\end{tabular}

\section{CONCLUSIONS}

Within this study, we outlined a concept to reduce the impact of surface plasmon polariton modes onto the power dissipation spectra of top-emitting OLED devices. For this purpose a low refractive index layer is spin coated as a HTL onto to the opaque metal bottom contact. Thus, the surface plasmon polariton excited at this metal dielectric interface (oSPP) is shifted to smaller in-plane wavenumbers, enhancing the outcoupling efficiency of the OLED. By using ultrathin metal electrodes, the area in phase space contributing to power dissipation is compressed. Thus the outcoupling efficiency of the device is enhanced even more. Experimentally the EQE for a low refractive index HTL OLED was enhanced by about $20 \%$ compared to the reference device. We were able to attribute this enhancement in EQE to the enhanced outcoupling efficiency of the device.

We point out that with an appropriate choice of material this concept can be adapted to bottom-emitting OLEDs comprising an opaque metal top contact. Simulations indicate that similar to top-emitting OLEDs with ultrathin top contacts, an enhancement of outcoupling efficiency for bottom-emitting OLEDs of about $20 \%$ is to be expected. Furthermore, the oSPPs dispersion relation can be shifted to in-plane wavenumbers smaller than $k_{0} n_{\text {active. }}$. Thus within the cavity the occurring fields can no longer be represented by an interface mode having a purely evanescent envelope in $z$-direction. In future work this may allow to extract the SPP contribution to the dissipated power, e.g. by using geometrical outcoupling techniques such as microlens structures.

\section{Acknowledgments}

This work was funded by the European Social Fund and the free state of Saxony through the OrganoMechanics project. Support from the excellence cluster cfaed is gratefully acknowledged. 


\section{Appendix}

a)

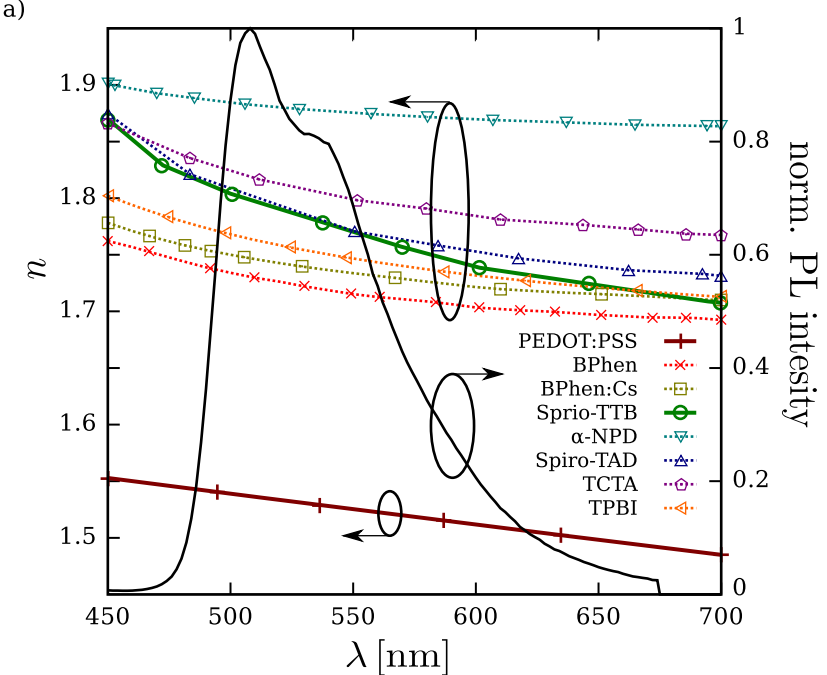

b)

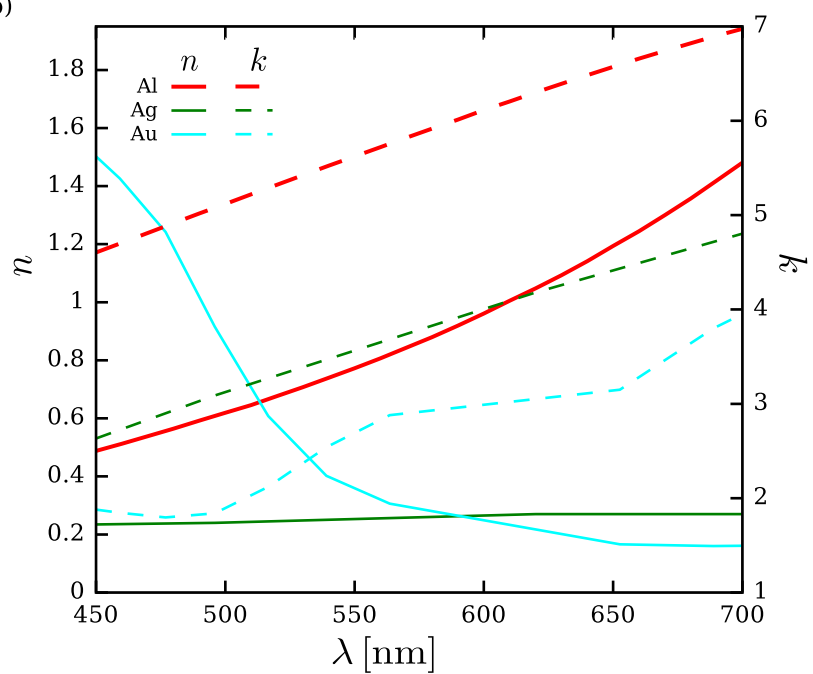

Figure 5. a) Real part $n$ of refractive index of dielectric materials used within simulations and normalized PL-intensity of green emitter. b) Real part $n$ and imaginary part $k$ of refractive index for the metals used in the simulation.

\section{REFERENCES}

[1] Hofmann, S., Thomschke, M., Lüssem, B., and Leo, K., "Top-emitting organic light-emitting diodes," Opt. Express 19(S6), A1250-A1264 (2011).

[2] Furno, M., Meerheim, R., Thomschke, M., Hofmann, S., Lüssem, B., and Leo, K., "Outcoupling efficiency in small-molecule OLEDs: from theory to experiment," in [Proc. SPIE 7617, Light-Emitting Diodes: Materials, Devices, and Applications for Solid State Lighting XIV], Streubel, K. P., Jeon, H., Tu, L.-W., and Linder, N., eds., Proc. SPIE (761716) (2010).

[3] Dakin, J. P., Bates, R., and Digonnet, M., "Optoelectronic Devices and Techniques," in [Handbook of Optoelectronics], Dakin, J. P. and Brown, R. G. W., eds., CRC Press Taylor \& Francis Group (2006).

[4] Patel, N. K., Cinà, S., and Burroughes, J. H., "High-efficiency organic light-emitting diodes," IEEE J. Sel. Top. Quant. Electron. 8(2), 346-361 (2002).

[5] Furno, M., Meerheim, R., Hofmann, S., Lüssem, B., and Leo, K., "Efficiency and rate of spontaneous emission in organic electroluminescent devices," Phys. Rev. B 85(11), 115205 (2012).

[6] Walzer, K., Maennig, B., Pfeiffer, M., and Leo, K., "Highly efficient organic devices based on electrically doped transport layers," Chem. Rev. 107(4), 1233-1271 (2007).

[7] Adachi, C., Baldo, M. A., Thompson, M. E., and Forrest, S. R., "Nearly 100\% internal phosphorescence efficiency in an organic light-emitting device," J. Appl. Phys. 90(10), 5048-5051 (2001).

[8] Reineke, S., Lindner, F., Schwartz, G., Seidler, N., Walzer, K., Lüssem, B., and Leo, K., "White organic light-emitting diodes with fluorescent tube efficiency," Nature 459, 234-238 (2009).

[9] Wang, Z. B., Helander, M. G., Qui, J., Puzzo, D. P., Greiner, M. T., Hudson, Z. M., Wang, S., Liu, Z. W., and Lu, Z. H., "Unlocking the full potential of organic light-emitting diodes on flexible plastic," Nat. Photonics 5, 753-757 (2011).

[10] Pitarke, J. M., Silkin, V. M., Chulkov, E. V., and Echenique, P. M., "Theory of surface plasmons and surface-plasmon polaritons," Rep. Prog. Phys. 70(1), 1-87 (2007).

[11] Sullivan, K. G. and Hall, D. G., "Enhancement and inhibition of electromagnetic radiation in plane-layered media. I.plane-wave spectrum approach to modeling classical effects," J. Opt. Soc. Am. B 14(5), 1149-1159 (1997).

[12] Sullivan, K. G. and Hall, D. G., "Enhancement and inhibition of electromagnetic radiation in plane-layered media. II. Enhanced fluorescence in optical waveguide sensors," J. Opt. Soc. Am. B 14(5), 1160-1166 (1997). 
[13] Revelli, J. F., Tutt, L. W., and Kruschwitz, B. E., "Waveguide analysis of organic light-emitting diodes fabricated on surfaces with wavelength-scale periodic gratings," Appl. Optics 44(16), 3224-3237 (2005).

[14] Hofmann, S., Thomschke, M., Freitag, P., Furno, M., Lüssem, B., and Leo, K., "Top-emitting organic light-emitting diodes: Influence of cavity design," Appl. Phys. Lett. 97, 253308 (2010).

[15] Meerheim, R., Furno, M., Hofmann, S., Lüssem, B., and Leo, K., "Quantification of energy loss mechanisms in organic light-emitting diodes," Appl. Phys. Lett. 97, 253305 (2010).

[16] Raether, H., [Surface Plasmons on smooth and rough surfaces and on gratings], Springer-Verlag Berlin Heidelberg New York London Paris Tokyo (1986).

[17] Raether, H., "Surface plasma oscillations and their applications," in [Physics of Thin Films], Hass, G., Francombe, M., and Hoffman, R., eds., 9, Academic Press New York (1977).

[18] Davis, T. J., "Surface plasmons modes in multi-layer thin films," Opt. Commun. 282(1), 135-140 (2009).

[19] Amra, C. and Maure, S., "Electromagnetic power provided by sources within multilayer optics: free-space and modal patterns," J. Opt. Soc. Am. A 14(11), 3102-3113 (1997).

[20] Webster, M. A., Auld, J. L., Martin, S. J., and Walker, A. B., "Simulation of the external quantum efficiency for bilayer organic light emitting devices," in [Proc. SPIE 5214, Organic Light Emitting Materials and Devices VII], Kafafi, Z. H. and Lane, P. A., eds., 300-309 (2004).

[21] Rigneault, H. and Monneret, S., "Modal analysis of spontaneous emission in a planar microcavity," Phys. Rev. A 54(3), 2356-2368 (1996).

[22] Schubert, S., Meiss, J., Müller-Meskamp, L., and Leo, K., "Improvement of Transparent Metal Top Electrodes for Organic Solar Cells by Introducing a High Surface Energy Seed Layer," Adv. Energy Mater. 3(4), 438-443 (2013).

[23] Schwab, T., Schubert, S., Hofmann, S., Fröbel, M., Fuchs, C., Thomschke, M., Müller-Meskamp, L., Leo, K., and Gather, M. C., "Highly Efficient Color Stable Inverted White Top-Emitting OLEDs with Ultra-Thin Wetting Layer Top Electrodes," Advanced Optical Materials 1(10), 707-713 (2013).

[24] Furno, M., Gather, M. C., Lüssem, B., and Leo, K., "Coupled plasmonic modes in organic planar microcavities," Appl. Phys. Lett. 100, 253301 (2012).

[25] Jönsson, S. K. M., Salaneck, W. R., and Fahlman, M., "X-ray photoelectron spectroscopy study of the metal/polymer contacts involving aluminum and poly(3,4-ethylenedioxythiophene)-poly(styrenesulfonic acid) derivatives," J. Mater. Res. 18(5), 1219-1226 (2003).

[26] Yamanari, T., Taima, T., Sakai, J., Tsukamoto, J., and Yoshida, Y., "Effect of buffer layers on stability of polymer-based organic solar cells," Jpn. J. Appl. Phys. 49(1), 01AC02-1-4 (2010). 